\title{
Study on the Preparation and Performance of APEG-AMPS-MA Polycarboxylate Superplasticizer
}

\author{
Jinming Wang \\ School of Chemical Engineering,Anhui University of Science and Technology, Huainan 232001, \\ China \\ email: wjm660088@163.com
}

\begin{abstract}
Keywords: polycarboxylate superplasticizer; fluidity; water-reducing rate; performance
Abstract. In this research, polycarboxylate superplasticizer is synthetized by solution polymerization. Some influencing factors on synthesis of the product are studied such as raw material composition, reaction time, initiator and $\mathrm{pH}$ value of the reaction system. The results show: When the amount of MA used in the reaction is $14 \%$, the fluidity and the water-reducing rate of the product reach the highest, $240 \mathrm{~mm}$ and 54\% respectively; when the amount of AMPS used in the reaction is 14\%, the fluidity and the water-reducing rate of the product reach the highest, $250 \mathrm{~mm}$ and $56.5 \%$ respectively; when the amount of the initiator used in the reaction accounts for $8 \%$, the fluidity and the water-reducing rate of the product reach the highest, $250 \mathrm{~mm}$ and $56.5 \%$ respectively; when the reaction time is 2 hours, the fluidity and the water-reducing rate of the product reach the highest, $230 \mathrm{~mm}$ and $53.5 \%$ respectively; when $\mathrm{pH}$ value is 2 , the fluidity and the water-reducing rate of the product reach the highest, $230 \mathrm{~mm}$ and $53.5 \%$ respectively.
\end{abstract}

\section{Introduction}

Polycarboxylate superplasticizer is an indispensable material in modern civil and architecture engineering as it embodies many important properties such as improving structure and strength of concrete, enhancing uniformity of the internal structure of concrete, reducing possible interface defects in concrete and improving corrosion resistance of concrete. Besides, it can improve the performance such as reducing water consumption, shortening clotting time of concrete, improving fluidity and workability of concrete, and thus the construction efficiency can be greatly enhanced. So such water-reducing agent has high water-reducing rate, low drainage rate, brings good workability and strength of concrete ${ }^{[1-4]}$. Different synthetic technologies and raw materials produce different types of polycarboxylate superplasticizer with varied properties, for example, early strength type, retarded type, high strength type and so on ${ }^{[7-8]}$. In this research, polycarboxylate superplasticizer is produced by copolymerization of maleic anhydride, acrylic polyethers and 2-acrylamido-2-methyl propane sulfonic acid in certain proportions, and its properties are investigated.

\section{Experiment}

\section{Instruments, chemicals and materials}

Experimental instruments: HL-2D digital constant flow pump; precision power electric blender JJ-1 100W; model HH-2 digital thermostatic water bath, FA2004N analytical balance (degree of accuracy: $0.1 \mathrm{mg}$ ); Jiming electronic balance; model NJ-160A cement paste mixer; DHG-9030B constant-temperature blast drying box

Chemicals and materials: sodium hydroxide, AR; 2-acrylamido-2-methyl propane sulfonic acid (AMPS), AR; maleic anhydride (MA), AR; acrylic polyethers (APEG), CP; ammonium persulfate (AKPS), AR; P.C32.5 cement

\section{Experimental Procedure}

Based on the principle of free radical reaction, the experiment uses ammonium persulfate as an initiator to create free radical polymerization reaction of 2-acrylamido-2-methyl propane sulfonic acid, and acrylic polyethers. The experiment procedure is as follows: 
(1) Measure a certain amount of maleic anhydride, 2-acrylamido-2-methyl propane sulfonic acid and acrylic polyethers and put them into a three-necked flask. Then put in some water and heat them in a thermostatic water bath until they dissolve totally in water. After that, use a pipette to add $20 \mathrm{ml}$ sodium hydroxide solution with a concentration of $20 \%$ to the flask.

(2) Add a certain amount of ammonium persulfate solution with a constant flow pump within a given time, and the reaction temperature rises to $55^{\circ} \mathrm{C}$ at the same time. When $1 / 3$ of the initiator has been added, the temperature rises to $75^{\circ} \mathrm{C}$. When another $1 / 3$ of the initiator has been added, the temperature rises to $85^{\circ} \mathrm{C}$. When the last $1 / 3$ of the initiator has been added, the temperature rises to $95^{\circ} \mathrm{C}$. One hour after the reaction, test the samples according to GB/T8077-2000 to get to know the fluidity of cement paste. Water cement ratio of the test is $0.29 \%$, and the water reducer content is $0.4 \%$.

\section{Results and Discussion}

\section{Influence of MA on the performance of polycarboxylate superplasticizer}

The experiment studies the change of polycarboxylate superplasticizer's performance by changing the proportion of the mass of MA to the total mass of APEG and AMPS while the ratio of the mass of APEG to the mass of AMPS is definite. The results of the experiment are shown in Table 1.

Table 1 MA's influence on the fluidity of cement paste of polycarboxylate superplasticizer

\begin{tabular}{lll}
\hline MA/\% & fluidity of cement paste/mm & water-reducing rate/\% \\
\hline 8 & 180 & 45.6 \\
11 & 230 & 53.5 \\
14 & 240 & 54.2 \\
17 & 235 & 53.7 \\
\hline
\end{tabular}

Table 1 shows: When the ratio of the mass of APEG to the mass of AMPS is fixed, with the increase in the amount of MA, the fluidity and the water-reducing effect of cement paste of polycarboxylate superplasticizer are enhanced at first and then decrease. If the proportion of MA is within 11\%-17\%, the fluidity and the water-reducing rate of the product are comparatively stable. They are within 230 240mm and 53.5\% 54.2\% respectively, which can meet the engineering utilization requirements. When the proportion of MA is $14 \%$, the fluidity and water-reducing effect reach their best, which are $250 \mathrm{~mm}$ and $56.5 \%$ respectively.

\section{Influence of AMPS on the performance of polycarboxylate superplasticizer}

The experiment studies the change of polycarboxylate superplasticizer's performance by changing the proportion of the mass of AMPS to the total mass of APEG and AMPS while the ratio of the mass of APEG to the mass of AMPS is definite. The results of the experiment are shown in Table 2.

Table 2 AMPS' influence on the fluidity of cement paste of polycarboxylate superplasticizer

\begin{tabular}{lll}
\hline AMPS/\% & fluidity of cement paste $/ \mathrm{mm}$ & water-reducing rate/\% \\
\hline 8 & 220 & 53.0 \\
11 & 230 & 53.5 \\
14 & 250 & 56.5 \\
17 & 230 & 53.5 \\
\hline
\end{tabular}

Table 2 shows: When the ratio of the mass of APEG to the mass of MA is fixed, with the increase in the amount of AMPS, the fluidity and the water-reducing effect of cement paste of polycarboxylate superplasticizer are enhanced at first and then decrease. If the proportion of AMPS is within $11 \%-17 \%$, the fluidity and the water-reducing rate of the product are good enough to meet the engineering utilization requirements. When the proportion of MA is $14 \%$, the fluidity and water-reducing effect reach their best, which are $250 \mathrm{~mm}$ and $56.5 \%$ respectively.

\section{Influence of initiator on the performance of polycarboxylate superplasticizer}

The experiment studies how the amount of initiator used in the reaction influences the performance of the polycarboxylate superplasticizer produced from the reaction system when the mass ratio of APEG, 
AMPS and MA, reaction time, and temperature are fixed. The results of the experiment are shown in Table 3.

Table 3 Initiator's influence on the fluidity of cement paste of polycarboxylate superplasticizer

\begin{tabular}{lll}
\hline initiator $/ \%$ & fluidity of cement paste $/ \mathrm{mm}$ & water-reducing rate $/ \%$ \\
\hline 6 & 240 & 54.2 \\
8 & 250 & 56.5 \\
10 & 200 & 48.8 \\
12 & 160 & 42.1 \\
\hline
\end{tabular}

Table 3 shows: When the mass ratio of APEG, AMPS and MA, reaction time, and temperature are fixed, with the increasing use of initiator, the fluidity and the water-reducing effect of cement paste of polycarboxylate superplasticizer are enhanced at first and then decrease. If the proportion of initiator is within 6\%-8\%, the fluidity and the water-reducing rate of the product are good enough to meet the engineering utilization requirements. When the proportion of initiator is $8 \%$, the fluidity and water-reducing effect reach their best, which are $250 \mathrm{~mm}$ and $56.5 \%$ respectively. Different amounts of initiator brings about noticeable change in the fluidity and the water-reducing rate of the product, so conclusion can be reached that the fluidity and the water-reducing effect of the product are sensitive to the amount of initiator used in the reaction under this condition.

\section{Influence of reaction time on the performance of polycarboxylate superplasticizer}

The experiment studies the influence of reaction time on the performance of the polycarboxylate superplasticizer produced from the reaction system under the condition that the mass ratio of APEG, AMPS and MA, initiator, and temperature are fixed. The results of the experiment are shown in Table 4

Table 4 Influence of reaction time on the fluidity of cement paste of polycarboxylate superplasticizer

\begin{tabular}{lll}
\hline reaction time/h & fluidity of cement paste $/ \mathrm{mm}$ & water-reducing rate $/ \%$ \\
\hline 1.5 & 220 & 53.0 \\
2 & 230 & 53.5 \\
2.5 & 225 & 53.2 \\
3 & 210 & 51.7 \\
\hline
\end{tabular}

Table4 shows: When the mass ratio of APEG, AMPS and MA, initiator, and temperature are fixed, with the increase in reaction time, the fluidity and the water-reducing effect of cement paste of polycarboxylate superplasticizer are enhanced at first and then decrease. If the reaction time is around $2 \mathrm{~h}$, the fluidity and the water-reducing rate of the product are good enough to meet the engineering utilization requirements. When the reaction time is exactly $2 \mathrm{~h}$, the fluidity and water-reducing rate reach their best, which are $230 \mathrm{~mm}$ and $53.5 \%$ respectively. Reaction time doesn't have significant influence on the fluidity and the water-reducing effect of cement paste of polycarboxylate superplasticizer, so a conclusion can be reached that the fluidity and the water-reducing effect of the synthetic product are not sensitive to reaction time under this condition.

\section{Influence of the $\mathrm{pH}$ value of reaction system on the performance of polycarboxylate superplasticizer}

The experiment studies how the change of the $\mathrm{pH}$ values of the reaction system influences the performance of the polycarboxylate superplasticizer when the mass ratio of APEG, AMPS and MA, initiator, reaction time and temperature are fixed. The results of the experiment are shown in Table 5.

Table 5 Influence of the $\mathrm{pH}$ value of reaction system on the fluidity of cement paste of polycarboxylate superplasticizer

\begin{tabular}{lll}
\hline $\mathrm{pH}$ value & fluidity of cement paste $/ \mathrm{mm}$ & water-reducing rate $/ \%$ \\
\hline 2 & 230 & 53.5 \\
6 & 200 & 48.8 \\
8 & 180 & 45.6 \\
10 & 160 & 42.1 \\
\hline
\end{tabular}

Table 5 shows: When the mass ratio of APEG, AMPS and MA, initiator, reaction time and temperature are fixed, with the increase in $\mathrm{pH}$ value of reaction system, the fluidity and the 
water-reducing effect of cement paste of polycarboxylate superplasticizer are on the decrease. When $\mathrm{pH}$ value of reaction system is low, the fluidity and the water-reducing effect of cement paste of the product are comparatively good enough to meet the engineering utilization requirements. When $\mathrm{PH}$ value is 2 , the fluidity and water-reducing rate reach their best, which are $230 \mathrm{~mm}$ and $53.5 \%$ respectively. So, under this condition, $\mathrm{PH}$ value of reaction system has significant influence on the fluidity and water-reducing effect of the product; acid environment helps to produce the product of better fluidity and water-reducing effect.

\section{Conclusions:}

From the above, the following conclusions can be reached:

(1) Under this proportion and other reaction conditions, the $\mathrm{pH}$ value of reaction system has the greatest influence on the fluidity and the water-reducing effect of polycarboxylate superplasticizer. The lower the $\mathrm{pH}$ value is, the better the fluidity and the water-reducing effect are.

(2) Under given conditions, the amount of MA used in the reaction has great influence on the fluidity and the water-reducing effect of the product. When the proportion of MA is within $11 \% \sim 17 \%$, the fluidity and the water-reducing effect of the product are all fairly good, but the ideal proportion is $14 \%$. Instead, the amount of AMPS used in the reaction only has mild effect on the fluidity and the water-reducing effect of the product, the ideal amount is $14 \%$.

(3) Under given conditions, the proportion of the amount of initiator has great influence on the fluidity and the water-reducing effect of the product. When the amount is within $6 \% \sim 8 \%$, the fluidity and the water-reducing effect of the product are all fairly good, but the ideal amount of initiator is $8 \%$.

(4) Under given conditions, reaction time also has relatively mild influence on the fluidity and the water-reducing effect of the product; the ideal reaction time is $2 \mathrm{~h}$.

\section{Acknowledgements:}

Supported by National Key Technologies R \& D Program of Huainan China(2013A4002)

\section{References}

[1] X. P. Lei: Bulletin of the Chinese Ceramic Society,2010,p. 1468. In Chinese

[2] X. P. Lei and Z. S. Feng: Bulletin of the Chinese Ceramic Society , 2013,p,2306. In Chinese

[3] X. M. Zhang, J. X. Lu and Y.L.Yu: Concrete,2010,p,104. In Chinese

[4] L. G. Xiao, C. Y. Yan and S. T. Zhang: Concrete,2013,p, 41. In Chinese

[5] S. Zhao, E. K. Fu, M. Li, et al. Concrete,2014,p,89. In Chinese

[6] Y.D.Xu, Q.Y.Zhao, X.P.Chen, et al. Concrete,2013,p,101. In Chinese

[7] G. B. Ma, J. B. Hu, H. B. Tan: New Building Materials,2012,p,1. In Chinese

[8] X. Y. Yin, C. H. Ou and Z. G. Zhang: Ready-mixed Concrete, 2014, p,37. In Chinese 